\title{
Expanding the Boundaries of Major Depressive Disorder in DSM-5: The Removal of the Bereavement Exclusion
}

\author{
H. Russell Searight \\ Department of Psychology, Lake Superior State University, Sault Sainte Marie, USA \\ Email: hsearight@lssu.edu
}

Received November $19^{\text {th }}$, 2013; revised January $3^{\text {rd }}$, 2014; accepted January $13^{\text {th }}$, 2014

\begin{abstract}
Copyright @ $2014 \mathrm{H}$. Russell Searight. This is an open access article distributed under the Creative Commons Attribution License, which permits unrestricted use, distribution, and reproduction in any medium, provided the original work is properly cited. In accordance of the Creative Commons Attribution License all Copyrights (c) 2014 are reserved for SCIRP and the owner of the intellectual property H. Russell Searight. All Copyright (c) 2014 are guarded by law and by SCIRP as a guardian.
\end{abstract}

\section{Introduction}

The recent publication of the Fifth Edition of the Diagnostic and Statistical Manual of Mental Disorders (DSM 5; American Psychiatric Association, 2013) appears to have triggered more controversy than its predecessors. The DSM 5, the first significant revision of this internationally accepted system for psychiatric diagnosis in the past 20 years, has been the subject of much pre- and post-publication criticism and debate-much of which has occurred in the popular press (Satel, 2013). One of these controversies centers on the distinction between major depressive disorder (MDD) and bereavement. Technically, "bereavement" refers to an individual's state after losing a loved one while "grief" describes the individual's psychological reaction to the loss. The two terms are often used interchangeably in the literature-a convention which will be followed herein.

Historically, previous DSM guidelines have included a proviso that clinicians should not diagnose a patient with MDD when there was evidence that the symptoms occurred immediately after the death of a loved one. Specifically, while the symptoms for MDD as well as their duration, have remained essentially the same in the transition from DSM-IV-TR to DSM-5, the guidelines for excluding a diagnosis of MDD in the context of bereavement have been removed in the recent revision. In the DSM-IV-TR, there was a clear directive at the end of the list of MDD's diagnostic criteria to avoid diagnosing MDD if symptoms were better accounted for by bereavement (American Psychiatric Association, 2000). In the previous system, even if the specific criteria and time duration had been fulfilled for a major depressive episode (MDE), the diagnosis was not given if symptoms were temporally associated with the death of a loved one and were of less than two months' duration. The rationale for the exclusion, though not well articulated in the DSM, has generally been assumed to avoid placing a medical diagnosis on a normal, albeit emotionally difficult, life transition. In addition, grief research had indicated that MDE symptoms and a bereavement-related reaction overlapped considerably (Wakefield, 2011). It was assumed that when associated with bereavement, depressive symptoms would dissipate in several months without formal treatment. These assumptions have been called into question-a factor likely associated with the recent bereavement exclusion.

While not specifically stated in the DSM 5, research evi- dence has accumulated over the past two decades calling the bereavement exclusion into question. Investigators have found few differences in symptoms between recently bereaved individuals and those with MDE (Horwitz \& Wakefield, 2007). Additionally, there have been a small series of studies indicating that bereaved individuals may benefit from antidepressant medication. While the DSM-5 includes an extended footnote about how to distinguish MDE from bereavement, the note concludes with an admonition to clinicians to be aggressive in diagnosing depression even in the context of a recent loss. Critics of the DSM-5 have suggested that the decision to drop the bereavement exclusion reflects the medicalization of distress, a growing norm in Western culture to translate psychosocial distress into medical symptoms with a pharmacological treatment. These critics view the removal of the bereavement exclusion as part of an economically fueled movement to expand the boundaries of psychopathology to increase the market for pharmacotherapy (Frances, 2013; Greenberg, 2013). From a broader philosophical perspective, there are concerns that bereavement-related distress may be meaningful for the survivors and should not be artificially diminished with psychopharmacologic balm (Frances, 2013; Elliott, 2000).

\section{DSM-5 Recommendations for Distinguishing Grief from MDD}

The DSM-5 contains an extended footnote about distinguishing grief from MDD. While acknowledging that dysphoriamay be part of grief, this mood state is seen as much more persistent and constant in MDD compared with bereavement. Grieving individuals are likely to experience more variability in mood including periods of happiness alternating with intense sadness triggered by thoughts of the deceased. The course of grief also differs with sadness becoming less intense in days to weeks while MDD's adverse mood states are much more extended. Worthlessness and diminished self-esteem, while common in MDD, are generally absent in grief. DSM-5 acknowledges that both MDD and bereavement may be associated with suicidal ideation; however, the underlying motivation differs. In MDD, suicidal thoughts are commonly associated with feelings of worthlessness or as a mechanism to relieve emotional suffering. By contrast, suicidal thinking is less common in bereavement and when it occurs is usually associated with a desire 
to join the deceased. The DSM-5 authors caution diagnosticians to be aware of the likelihood of MDD even in the context of interpersonal loss.

While some of these distinguishing criteria seem relatively clear, the ability to discriminate bereavement's "feelings of emptiness and loss" from MDE's "persistent depressed mood and ...inability to anticipate happiness or pleasure" (American Psychiatric Association, 2013; p. 161), is likely to demand more of the clinician's acumen and time. Given that most patients with MDD are diagnosed and treated by primary care physicians (Callahan \& Berrios, 2005), it may be difficult to distinguish these subtleties in a standard 10 - 15 minute office visit particularly when there are comorbid medical issues.

\section{Bereavement Does Not Differ from Reactions to Other Life Stressors}

The removal of the bereavement exclusion in the DSM- 5 has been attributed to several research findings in the past 20 years. Historically, the DSM, while not making it a formal diagnosis, has included discussion of bereavement. For example, DSMIV-TR describes the difference between complicated bereavement which triggers an episode of MDD and symptoms associated with loss that resolve within two months which are not formally diagnosed (American Psychiatric Association, 2000). Wakefield and colleagues completed a series of studies and concluded that there is little support for the bereavement exclusion. Comparisons of persons fitting DSM IV-TR's description of complicated bereavement found little difference in symptoms or severity of symptoms from those with uncomplicated bereavement (Wakefield, Schmitz, First, \& Horwitz, 2007). Unlike MDD which is often chronic, only $10 \%-12 \%$ of persons who are bereaved still show symptoms of MDD at one year following the loss (Wakefield \& Horwitz, 2007). It is estimated that without the bereavement exclusion, approximately $20 \%$ $40 \%$ of bereaved individuals would be diagnosed with MDD (Horwitz \& Wakefield, 2007).

Wakefield et al., (2007) provide additional evidence supporting the view that the MDD's diagnostic boundaries should be contracted rather than expanded. In comparative analyses of large samples of persons with depressive symptoms, they found no differences in the actual symptoms or their severity in response to death of a loved one versus in response to other losses such as sudden unemploymentor, marital dissolution. Based upon these findings, Wakefield and colleagues (2007) have argued that when MDD symptoms are associated with any type of significant life event, a mood disorder diagnosis should not be given. Wakefield's (2011) major criticism of the DSM criteria is that they fail to take into account the symptoms' context and the bereavement exclusion should be extended to emotional reactions to these other life events-thus reducing the prevalence of MDD.

Conversely, however, these research findings and the accompanying reasoning can be used to support the view that bereaved individuals should receive a diagnosis of MDD. Wakefield and colleagues found that the same complement of depressive symptoms characterizing bereavement were no different than symptomatic responses to other life events such as anticipated work lay-off or learning of a romantic partner's infidelity (Horwitz \& Wakefield, 2007). Since individuals experiencing depressive symptoms associated with other life stressors would, according to current standards, be diagnosed with MDD if symptoms were present for two weeks, bereaved individuals should receive the diagnosis as well. With the removal of the bereavement exclusion, Horwitz and Wakefield (2007) predict that one-third to one-half of bereaved individuals will meet criteria for MDD during the first month immediately following the loss

\section{Pharmacotherapy of Bereavement}

Treatment of grief with psychotropic medication has been studied in small samples-often without the benefit of double blind placebo controlled designs. Another limitation of this research is that the conditions treated in these studies are somewhat heterogeneous with complicated grief, bereavement-related MDD and bereavement alone included. Early studies, using tricyclic antidepressants (TCAs) demonstrated some reduction in depressive symptoms but a more modest effect for grief intensity. While the majority of studies are simple prepost investigations with small sample sizes, Zisook and colleagues (2001) compared the effects of an 8 week trial bupropion on a group of patients compared with an intention-to-treat group. Both groups demonstrated significant improvement in depressive symptoms with a more modest reduction in grief. In a comparison of psychotherapy with and without medication, Reynolds and colleagues (1999) found the most favorable outcome on depressive symptoms for combined interpersonal psychotherapy and nortiptylene (69\%) with nortiptylene alone (56\%) demonstrating superiority over placebo groups. However, neither of these active treatment groups demonstrated significant reductions in grief intensity.

Studies involving SSRIs while fewer, suggest that these medications may have greater impact on grief as well as depression. For example, in an open-label trial of escitalopram, response to medication was $83 \%$ for depressive symptoms but only $45 \%$ for symptoms of complicated grief (Hensley, Slonominski, Uhlenhuth, \& Clayton, 2009). A smaller trial of 16 weeks of escitalopram found a $38 \%$ reduction in grief intensity after 16 weeks. Of note, however, the corresponding intention to treat group demonstrated a $24 \%$ reduction in grief (Bui, NadalVicens, \& Simon, 2012; Shear, Fagiolini, Houck, et al., 2006).

In sum, while not an exhaustive review of research in this area, findings to date suggest that medication for bereavement may lead to more rapid resolution and/or substantially reduce depressive symptoms. However, from a quantitative perspective, pharmacotherapy appears to have less pronounced impact on the experience of grief.

\section{Is Grief Necessary?}

Arguing that bereavement is not a psychiatric disorder, Frances (2013) and others have noted that grief is a common behavioral reaction that occurs among non-human mammals. For example, macaques deprived of group membership exhibited a stereotypic behavior pattern that differed from those with normal social contact. Of particular interest was the finding that these socially deprived macaques exhibited diminished serotonin activity in the prefrontal brain region (Bui, Nadal-Vinces, \& Simmon, 2012; Fontenot, Kaplan, Manuck, Arange, \& Mann, 1995). Evolutionary psychologists have generated multiple theories about grief. Outward changes in behavior may elicit support from others. Archer (1999) suggests that grief is the necessary outgrowth of the evolutionary advantage of social attach- 
ment.

While noting the commonality of the grief response across species, Frances (2013) also raises moral and philosophical objections to diagnosing grieving individuals with MDE. Frances argues that there is something inherently offensive in reducing grief to a disease: "Medicalizing grief reduces the dignity of the pain, short-circuits the expected existential processing of the loss, reduces reliance on the many well-established cultural rituals for consoling grief, and would subject grievers to unnecessary and potentially harmful medication” (Frances, 2013: p. 187). Medicalizing grief both impugns the integrity and "dignity" of the survivors' emotional experience, but also is disrespectful to the life that was lost (Frances, 2013). Grief and bereavement rituals are long-standing responses that have important meaning in their specific culture. In some cultures such as Japan, intense contemplation and melancholia have been seen as signs of morally superior character (Kitanaka, 2012). MDE, as a disorder is only beginning to be recognized in Japan (Kitanaka, 2012).

\section{Treating Grief: Harm or Enhancement}

The implicit corollary of converting grief to MDD is that within American medicine, diagnoses are inextricably tied to available treatment. However, if bereavement can be treated with pharmacotherapy, should it be?

Before further discussion of the moral side of this issue, it is worthwhile to consider the impact of implicitly diagnosing and overtly treating everyone exposed to a stressful live event. Critical Incident Stress Debriefing (CISD), typically administered as a group intervention, to persons exposed to life-threatening traumatic events such as first responders, continues to be commonly used, and often mandated. However, data from multiple studies suggest that the iatrogenic affects often outweigh any benefit from CISD (Lohr, Hooke, Gist, \& Tolin, 2004). While there are likely multiple explanations for this finding, it is likely that many first responders have a working coping style, often including some element of avoidance, that is successful. By forcing these individuals to repeatedly relive the trauma and face the accompanying emotional turmoil, successful adaptation may be prevented. Research on CISD suggests that providing psychological treatment to all who experience a traumatic event may actually harm those receiving it. Wakefield (2011) notes that similar to PTSD, the meaning of bereavement's depressive symptoms depend on the context in which they occur. Re-defining bereaved individuals as ill subject them to unwanted treatment that may challenge pre-existing coping skills.

However, if a medication can reduce symptoms and improve functioning, should everyone losing a loved one be required to simply “muddle through" (Horwitz \& Wakefield, 2007: p. 23) life's inherent complications when there is a relatively convenient pharmacological alternative? Aside from the possible side effects of antidepressants and the finding that $30 \%-40 \%$ of patients prescribed these medications fail to improve, is there potential harm from labeling all recently bereaved individuals as psychiatric patients? As a clinician, the author remembers the days before SSRIs when tricyclic antidepressants (TCAs) were commonly prescribed. While SSRI's are not free of side effects, they are not usually as disruptive as the pronounced sedation, and anticholinergic effects during the first 7 - 10 days of taking a TCA. The question remains-if there are few ad- verse medication effects and the patient appreciates the possibility of being a non-responder, is there any reason not to be treated for bereavement?

If grief is seen as having little value and as an unfortunate life event that temporarily impairs functioning, the availability of pharmacotherapy to aid in coping should be welcome. Similar to cognitive enhancement with drugs such as Modafanil which extend concentration, antidepressant medication can reduce some of the distress accompanying bereavement. Critics of psychiatric enhancement are often described as espousing "pharmacological Calvinism" (Klerman, 1972), a view that difficulties in cognitive-emotional functioning are meaningful, character-building burdens to be shouldered rather than attenuated with psychotropic medication. Calvinism in particular, is relevant when it comes to bereavement. Medication may "cheapen" the experience of grief by making it less intense and disruptive to one's life. In some cultures, an individual demonstrating little sadness after the loss of a parent or spouse would be considered deviant because of the absence of extended mourning. Contemporary mental health Calvinists, argue that it is immoral to feel "good" after the loss of a loved one. Indeed, even in industrialized countries such as the US, there is concern that getting back to "normal " too soon is a form of denial and will be associated with a high level of unresolved grief or delayed emotional upheaval.

\section{Conclusion}

This essay has reviewed the clinical, empirical, and philosophical issues raised by both proponents and opponents of the DSM-5 bereavement exclusion. Whether widespread clinical application of DSM-5's bereavement exclusion will increase the incidence of MDE diagnoses remains to be seen. In addition, how readily patients will seek and accept pharmacotherapy to address the grief of interpersonal loss is also an open question. The "ground work" for pharmacotherapy of bereavement has been laid with the use of SSRIs to "buff up" one's personality (Kramer, 1993), and drugs such as modafanil to improve cognitive functioning and eliminate fatigue associated with shift work. However, bereavement, with its often specific cultural and religious context, does not appear comparable to these other uses of enhancement therapy. The ethical issues surrounding "artificial" coping with loss through the medicalization of bereavement are likely to continue to be debated.

\section{REFERENCES}

American Psychiatric Association (2000). Diagnostic and statistical manual of mental disorders. DSM-IV-TR (4th ed., text revision). Washington DC: American Psychiatric Association.

American Psychiatric Association (2013). Diagnostic and statistical manual of mental disorders. DSM-5 (5th ed.). Washington DC: American Psychiatric Association.

Archer (1999). The nature of grief: The evolution and psychology of reactions to loss. London: Routledge.

Bui, E., Nadal-Vicens, M., \& Simon, N. M. (2012). Pharmacological approaches to the treatment of complicated grief: Rationale and a brief review of the literature. Dialogues in Clinical Neuroscience, 14, 149-157.

Callahan, C. M., \& Berrios, G. E. (2005). Reinventing depression: A history of the treatment of depression in primary care 1940-2004. New York: Oxford.

Elliott, C. (2000). Pursued by happiness and beaten senseless: Prozac and the American dream. Hastings Center Report, 30, 7-12. 


\section{H. R. SEARIGHT}

http://dx.doi.org/10.2307/3528306

Fontenot, M. B., Kaplan, J. R., Manuck, S. B., Arango, V., \& Mann, J. J. (1995). Long-term effects of chronic social stress on serotonergic indices in the prefrontal cortex of adult cynomolgus macaques. Brain Research, 705, 105-108. http://dx.doi.org/10.1016/0006-8993(95)01146-3

Frances, A. (2013). Saving normal. New York: Harper Collins.

Greenberg, G. (2013). The book of woe: The DSM and the unmaking of psychiatry. New York: Penguin.

Hensley, P. L., Slonimiski, C. K., Uhlenhuth, E. H., \& Clayton, P. J. (2009). Escitalopram: An open-label study with bereavement-related depression and grief. Journal of Affective Disorders, 113, 142-149. http://dx.doi.org/10.1016/j.jad.2008.05.016

Horwitz, A. V., \& Wakefield, J. C. (2007). The loss of sadness: How psychiatry transformed normal sorrow into depressive disorder. New York: Oxford.

Kitanaka, J. (2012). Depression in Japan: Psychiatric cures for a society in distress. Princeton, NJ: Princeton University Press.

Klerman, G. L. (1972). Psychotropic hedonism vs. pharmacological Calvinism. Hastings Center Report, 3-4.

Kramer, P. (1993). Listening to Prozac: A psychiatrist explores antidepressant drugs and the remaking of the self. New York: Viking.

Lohr, J. M., Hooke, W., Gist, R., \& Tolin, D. F. (2003). Novel and controversial treatments for traumatic-related stress disorders. In S. Lilienfeld, S. Lynn, \& J. Lohr (Eds.), Science and pseudoscience in clinical psychology. New York: Guilford.

Reynolds, C. F., Miller, M. D., Pasternak, R. E., et al. (1999). Treatment of bereavement-related major depressive episodes in later life: A controlled study of acute and continuation treatment with nortriptylene and interpersonal psychotherapy. American Journal of Psychiatry, 156, 202-208.

Satel, S. (2013). Why the fuss over the DSM-5? New York Times. http://www.nytimes.com/2013/05/12/opinion/sunday/why-the-fuss-o ver-the-dsm-5.html? $\mathrm{r}=0$

Shear, M. K., Fagiolini, A., Houck, P., et al. (2006). Escitalopram for complicated grief: A pilot study. NCDEU 46th Annual Meeting Abstracts, Boca Raton, FL: National Institute of Mental Health.

Wakefield, J. C. (2011). Should uncomplicated bereavement-related depression be reclassified as a disorder in the DSM-5? The Journal of Nervous and Mental Disease, 199, 203-208. http://dx.doi.org/10.1097/NMD.0b013e31820cd155

Wakefield, J. C., Schmitz, M. F., First, M. B., \& Horwitz, V. (2007). Extending the bereavement exclusion for major depression to other losses. Archives of General Psychiatry, 64, 433-440. http://dx.doi.org/10.1001/archpsyc.64.4.433

Zisook, S., Shuchter, S. R., Pedrelli, P., Sable, J., \& Deacluc, S.C. (2001). Bupropion sustained release for bereavement: Results of an open trial. Clinical Psychiatry, 62, 227-230.

http://dx.doi.org/10.4088/JCP.v62n0403 